\title{
Diagnosis and management of restless legs syndrome in
}

\section{children}

\author{
Narong Simakajornboon ${ }^{a}$, Leila Kheirandish-Gozal ${ }^{\mathrm{b}}$, and David Gozal ${ }^{\mathrm{b}}{ }^{*}$ \\ a Cincinnati Children's Hospital Medical Center, Cincinnati, OH, USA \\ b Kosair Children Hospital Research Institute, Division of Pediatric Sleep Medicine, University of \\ Louisville, 570 S. Preston Street, Suite 204, Louisville, KY 40202, USA
}

\section{Summary}

Recent published evidence suggests that restless legs syndrome (RLS) and periodic limb movement disorder (PLMD) are common condition in children and adolescents. It is likely that if left untreated, RLS and PLMD may lead to adverse cardiovascular and neurocognitive consequences. However, the diagnosis of RLS and PLMD in children is challenging, particularly because children are relatively unable to describe typical RLS symptoms. The International Restless Legs Study Group has recently published consensus criteria for the diagnosis of RLS and PLMD in children. In addition to clinical description of RLS symptoms, supportive evidence including the presence of clinical sleep disturbances, documented periodic limb movements in sleep from overnight sleep study and family history of restless legs syndrome may be required. Few if any controlled studies have addressed the management of RLS and PLMD, which may involve both non-pharmacologic and pharmacologic approaches. In this context, the importance of avoidance of aggravating factors and good sleep hygiene cannot be overemphasized. Children with evidence of low-iron storage, i.e., low-serum ferritin and/ or iron levels may likely benefit from iron therapy. While there is overall limited experience regarding the use of dopaminergic agents in children with RLS and PLMD, published reports suggesting efficacy of compounds such as levodopa, ropinirole, pramipexole and pergolide have emerged. Other medications including benzodiazepine, anti-convulsants, alpha-adrenergic and opioid medications have not been adequately studied in children. Children with RLS and PLMD should have regular follow-up visits to evaluate clinical improvement and to monitor adverse effects from the selected therapy. Based on aforementioned findings, it is clear that a substantial research effort is needed to evaluate the pathophysiology,

\section{Keywords}

Restless legs syndrome; Periodic limb movement disorder; Dopaminergic medications; Iron therapy; Children; Attention-deficit hyperactivity disorder

\section{Introduction}

Restless legs syndrome (RLS) is a sensorimotor disorder characterized by the prototypic complaint of a strong, nearly irresistible urge to move the legs. Periodic limb movements in sleep (PLMS) are characterized by periodic episodes of repetitive and highly stereotypic

*Correspondence to: David Gozal, Department of Pediatrics, Comer Children's Hospital, The University of Chicago, 5721 S. Maryland Avenue, MC 8000, Suite K-160, Chicago, IL 60637, USA. Tel.: +1 (773) 834 1483; fax: +1 (773) 7024523. dgozal@peds.bsd.uchicago.edu (D. Gozal). 
limb movements during sleep. ${ }^{1}$ Periodic limb movement disorder (PLMD) is defined as the presence of periodic limb movement during sleep associated with symptoms of insomnia or excessive daytime sleepiness. RLS and PLMD are closely related. Most patients with PLMD do not manifest RLS symptoms; however, approximately $80 \%$ of adult RLS and $63-74 \%$ of pediatric RLS cases have PLMS. ${ }^{2,3,4}$

While most of the literature on RLS and PLMD has addressed the adult population, RLS and PLMD have been reported in children. In fact, careful history reveals that in $40 \%$ of adults suffering from RLS, the initial onset of symptoms will occur before 20 years of age. , $^{2,5}$ Unfortunately, it was only until very recently that the initial epidemiological study of RLS was reported in children. ${ }^{6}$ Based on this particular large scale survey, it appears that RLS is common in children and adolescents with an estimated prevalence of $1.9 \%$ in school aged children and $2 \%$ in adolescents in whom there were no significant gender differences. ${ }^{6}$ Such figures, if confirmed by additional studies, would indicate that approximately 984,000 children are affected by RLS in the United States alone.

The etiology of pediatric RLS and PLMD is currently poorly understood. It remains unclear as to which specific role(s) are played by genetic factors, dopamine dysfunction, and lowiron stores in the pathophysiology of RLS and PLMD. A recent study indicated the presence of a significant association between RLS and PLMD and a common variant in an intron of BTBD9 on chromosome 6p21.1, emphasizing the potential for both genetic predisposition and genetic susceptibility to the occurrence of RLS and PLMD. ${ }^{7}$ In addition, ethnic differences may also be present. ${ }^{49}$ There is also a significant proportion of shared comorbidity between Attention-deficit hyperactivity disorder (ADHD) and RLS and PLMD, implying the potential interactions between factors involved in the pathogenesis of ADHD and those underlying the onset and evolution of RLS and PLMD. ${ }^{50}$ However, despite substantial evidence pointing to a critical role for iron in adult with RLS and PLMD, we are unaware of specific studies that have addressed these issues in children.

The diagnosis of RLS in children can be quite challenging due to their inability to recognize or verbalize the presence of classic RLS symptoms. The International Restless Legs Study Group has published a set of proposed consensus criteria for reaching the diagnosis of RLS and PLMD in pediatric populations. ${ }^{8}$ Although RLS is a clinical diagnosis, supportive evidence such as presence of PLMS or family history of RLS, may be required in children. To further compound the already heightened level of uncertainty, the guidelines from the Standard of Practice Committee of the American Academy of Sleep Medicine state that no specific recommendations can be made regarding treatment of children with RLS and PLMD. ${ }^{1}$ However, since most children with RLS and PLMD may have low-iron stores, they may benefit from iron therapy, and any decision regarding use of other pharmacological agents will have to follow arbitrary and individualized algorithms, none of which has thus far sustained critical scientific validation. This paper will therefore proceed with a literature review on the diagnostic criteria and management of RLS and PLMD in children, with the understanding that few if any of the findings and potential recommendations emanating from such review are constrained by scientific scrutiny.

\section{Diagnostic criteria in children and adolescents}

The process of reaching the diagnosis of RLS in children is fraught with substantial challenging issues, particularly because young children may not be able to recognize and describe typical RLS symptoms or because these symptoms may not become manifest at very young ages. Indeed, the interval between the initial sleep consultation and the diagnosis of definite RLS revolves around 4.4 years. ${ }^{9}$ In addition, the period of time elapsing between onset of clinical sleep disturbances and the diagnosis of definite RLS is 11.6 years. ${ }^{9}$ 
Furthermore, RLS is under-recognized and therefore under-diagnosed, even among children whose family members seek medical advice for moderate to severe symptoms. ${ }^{6}$ As the result of an expert consensus workshop at the National Institutes of Health, the International Restless Legs Syndrome Study Group developed a set of criteria deemed necessary and sufficient for reaching a putative diagnosis of RLS and PLMD in children and adolescents. ${ }^{8}$ The certainty level of the diagnosis was classified into three categories, namely definite RLS, probable RLS, and possible RLS (Tables $1^{-} 3$ ). Because the diagnostic criteria for RLS in children are still evolving, the probable and possible RLS categories are intended for research purposes only in children aged 0 through 18 years, with the intent to capture the full spectrum of disease. ${ }^{8,10}$ For clinical purposes, the definite RLS criteria should be used for children aged 2 through 12 years old, while for adolescents (>12 years old), the adult diagnostic criteria are considered more appropriate. The definite RLS criteria in children are more stringent than those implemented for adults, and essentially aim to avoid over diagnosing RLS in children (see Table 1). In addition to the 4 essential adult criteria, children should be able to describe in their own words a set of sensations that would be consistent with leg discomfort. Other supportive evidence that can be used as part of the diagnostic process, includes the presence of sleep disturbance for age, a family history of RLS, and a polysomnographic finding of PLMS (PLM index $>5 / h$ ). The diagnostic criteria for PLMD in children are shown in Table 4. Symptoms of sleep disturbance are deemed essential for the diagnosis of PLMD in children. In addition, exclusion of other potential causes of PLMS, such as sleep-disordered breathing or medication-related side effects, is a requisite for the accurate diagnosis of PLMD. Although RLS and PLMD in adult population are separate entities, the relationship between RLS and PLMD in children is somewhat complex. The presence of PLMS is part of the supportive evidence for diagnosis of RLS in children. RLS is unlikely to be diagnosed prior to 5-6 years of age due to insufficient language, and cases of PLMD developing into RLS have been described. In fact, many children with definite RLS have been previously diagnosed with PLMD or met the research criteria for probable and possible RLS. ${ }^{9}$

The differential diagnosis includes other conditions associated with leg discomfort such as nocturnal leg cramps, arthritis, Osgood-Schlatter, neuropathies, and various types of dermatitis. ${ }^{8,10}$ In addition, several medical conditions can be associated with RLS including pregnancy, iron deficiency, renal failure, and children receiving dialysis. ${ }^{11}$ Another important differential diagnosis is positional discomfort which can superficially meet all the criteria for RLS. If a patient says that their discomfort is only in the left hip at night, when they lie down and that they get relief immediately by moving sideways to lie on their right hip, this is positional discomfort, and should not be viewed as RLS. In this sense, it is important to ask for the typical topographic distribution of RLS symptoms in taking the history. Although true RLS can exist in almost any part of the body, the typical distribution is in the thighs and calves. The diagnostic approach should begin with a thorough and complete clinical history. The characteristic RLS symptoms include the urge to move the legs or unpleasant sensations, which are worse at night and at rest, and are partially or totally relieved by movement. ${ }^{8}$ It is important to allow young children to give their own descriptions, and also provide them with well directed questions on such symptoms, such as to avoid introduction of bias. On physical examination, most children will have normal findings. A complete neurological examination is essential to rule out other causes of leg discomfort, such as neuropathy. In children, an overnight polysomnographic study documenting PLMS is crucial when classic RLS symptoms are absent. Because most children with RLS and PLMD have evidence of low-iron stores, it is important to obtain iron profiles including complete blood count, serum iron, and serum ferritin. Indeed, the role of iron metabolism in RLS and PLMD has emerged as an important determinant of disease severity and response to dopaminergic therapy. ${ }^{12}$ Furthermore, it remains unclear whether children with RLS and PLMD will display the same iron metal receptor alterations (i.e., 
transferrin receptor and divalent metal transporter 1 protein) in lymphocytes that have been documented in adults with the disease. ${ }^{13}$ Any children with suspected neuropathy should have additional tests including thyroid function, fasting blood sugar and insulin, and serum levels of vitamins B6, B9, and B12.14

\section{Consequences}

As in sleep-disordered breathing, RLS and PLMD can lead to both cardiovascular and neurocognitive consequences. Several recent studies have shown that RLS and PLMD in the adult population is associated with a rise in nocturnal blood pressure and hypertension. ${ }^{15,16}$ The mechanism underlying blood pressure changes may be related to autonomic activation in the context of repeated arousals. ${ }^{17}$ Currently, there are no data available on the cardiovascular consequences of RLS and PLMD in children. Cognitive deficits and ADHD have been reported in adults with RLS and PLMD. ${ }^{18,19}$ Several studies have demonstrated the association between RLS and PLMD and ADHD in children. ${ }^{20-25}$ The relationship between ADHD and RLS and PLMD can be explained by several possibilities. ${ }^{24}$ Firstly, sleep disruption associated with RLS and PLMD may lead to inattentiveness and hyperactivity. Secondly, RLS and PLMD may be a co-morbidity of ADHD. Thirdly, RLS and PLMD and subset of ADHD may share common dopamine dysfunction or alterations in iron metabolism. ${ }^{26-30}$ Finally, diurnal manifestations of RLS and PLMD may mimic ADHD. $^{24}$

Another co-morbidity of RLS and PLMD consists of the common parasomnias, such as confusional arousals, night terrors, sleepwalking, and nightmares. Several studies have shown that there is an increased frequency of parasomnias in children with RLS and PLMD. 21,31 The co-existence of RLS and PLMD and parasomnias and the resolution of parasomnias after treatment of RLS and PLMD suggest that sleep disruption associated with RLS and PLMD may trigger or facilitate the appearance of parasomnias. ${ }^{32}$

\section{Management}

\section{Non-pharmacologic}

It is important to identify medications or other factors that could aggravate RLS and PLMD and examine ways of discontinuing these medications. For instance, selective serotonin reuptake inhibitor (SSRI), metoclopramide, diphenhydramine, sleep deprivation, nicotine, caffeine, and alcohol have all been shown to either promote or aggravate RLS and PLMD.

10,14 Parents should be advised to avoid caffeine in these children. Regular sleep routines and good sleep hygiene are essential for the management of RLS in children. ${ }^{10,14}$ Sleep hygiene practices that should be encouraged include regular sleep and wake schedule, avoidance of heavy exercise and large meals close to bedtime, limiting exposure to bright light at night, and eliminating stimulating activities at night.

\section{Pharmacologic}

There is limited information regarding medical therapy in children with RLS and PLMD. The guideline from the Standard of Practice Committee of the American Academy of Sleep Medicine states that no specific recommendations can be made regarding treatment of children with RLS or PLMD. A recent population survey has shown that only $6.2 \%$ of children and $6.4 \%$ of adolescents with definite RLS received ongoing prescription medications. Furthermore, only $1.5 \%$ of patients received appropriate medications for RLS treatment based on the adult literature. ${ }^{6}$

Children with low-iron stores as defined by low-serum ferritin levels may benefit from iron therapy. Several studies have suggested the benefit of raising serum ferritin above $50 \mathrm{ng} / \mathrm{ml}$. 
27,33 The dose of iron therapy is $3 \mathrm{mg}$ of elemental iron $/ \mathrm{kg} /$ day. The duration of treatment used in our previous study was 3 months followed by slow tapering of the dose for a period of one year. The preliminary long-term follow-up of these children treated with iron therapy showed consistent evidence of sustained clinical improvements 1-2 years after iron therapy, with serum iron and ferritin remaining at adequate levels. ${ }^{34}$ Iron therapy seems to lead to long lasting improvement in clinical symptoms, and in our opinion, should be considered as the initial option, when serum ferritin levels are $<50 \mathrm{ng} / \mathrm{ml}$.

Dopaminergic medications are widely used and considered as the first line of treatment in adult patients with RLS and PLMD. ${ }^{35,36}$ Carbidopa/levodopa and dopamine agonists (pramipexole, ropinirole) are 2 commonly used dopaminergic medications in adult populations. Ropinirole was the first Food and Drug Administration (FDA)-approved medication for treatment of moderate to severe primary RLS as of May 2005. ${ }^{37}$ Subsequently, pramipexole was approved for the same indication in November 2006. The potential side effects of dopaminergic medications include nasal congestion, nausea, vomiting, insomnia, daytime sleepiness, hallucinations, obsessive-compulsive behavior, and fluid retention. ${ }^{14}$ Nausea is common with levodopa, but not with non-ergot dopamine agonists. The use of pergolide (an ergot dopamine agonist) has been reported to be associated with cardiac valve fibrosis. ${ }^{38}$ Worsening of RLS symptoms after initial improvement should raise the possibility of the phenomenon known as "augmentation". These patients usually manifest earlier symptoms during the day. Augmentation frequently occurs in patients using levodopa but can occur with any of the dopamine agonists. Augmentation can occur in 70-80\% of patients using daily levodopa and in $15-40 \%$ of those using dopamine agonists. ${ }^{36,39,40}$ The management of augmentation involves reducing dose, withdrawing dopaminergic medication and switching to another type of medication. ${ }^{35}$ One study has suggested that augmentation is associated with low-ferritin level. ${ }^{12}$

There is limited experience regarding the use of dopaminergic medications in children. Although ropinirole and pramipexole are FDA approved medications in adults with RLS and PLMD, there is no approved medication for the pediatric population. Published case reports show the effectiveness of levodopa, ${ }^{41}$ ropinirole,${ }^{42}$ pramipexole,${ }^{32}$ and pergolide ${ }^{41}$ in young children and adolescents. Levodopa and dopamine agonists result in long-term

improvements in children with RLS and PLMD. 9,42,43 The use of dopaminergic medications is associated with an improvement in RLS symptoms and reduction of PLMS and associated arousals. ${ }^{41}$ In children with ADHD and RLS and PLMD, the use of dopaminergic medications can result in improvements and even resolution of ADHD symptoms. ${ }^{41,42}$

Other medications including benzodiazepines, anti-convulsants, alpha-adrenergic, and opioid medications have not been adequately studied in children. Clonazepam is commonly used for treatment of RLS and PLMD in children. However, it may aggravate hyperactivity in children with ADHD. ${ }^{10}$ Gabapentin has been shown to reduce RLS symptoms and improve sleep quality. ${ }^{44,45}$ Clonidine is commonly used for children with sleep-onset problems and can be effective in children with RLS and PLMD. ${ }^{46}$

Children with RLS and PLMD should have regular follow-up visits to monitor clinical symptoms and to adjust the dose of medication as needed. A wide range of optimal doses for dopaminergic medications has been reported. ${ }^{10}$ Children receiving iron therapy should be periodically reassessed for their serum iron and ferritin, and the dose of iron supplement gradually adjusted to achieve the desired normalization of serum ferritin and iron levels. Since genetic factors play an important role in RLS and PLMD, parents may be affected and should be referred for further evaluation and treatment. However, we are unaware of specific genetic studies in children with RLS and PLMD. 


\section{Prognosis}

Currently, there is limited information on the long-term consequences and outcomes associated with RLS and PLMD in children. In adults with early onset RLS, there is a slow progression of the disease along with long periods of stability. A small percentage of patients can have a period of remission. ${ }^{5,47,48}$ In children, the same pattern of slow progression has been reported. ${ }^{9}$

\section{Practice points}

1. RLS and PLMD are common in children and adolescents.

2. There is a strong association between RLS and PLMD and ADHD.

3. Diagnosis of RLS and PLMD requires specific criteria. In addition to RLS symptoms, other supportive evidences such as the presence of sleep disturbances, documented PLMS and family history RLS are important.

4. Both pharmacologic and non-pharmacologic interventions are important in the management of RLS and PLMD in children.

5. Children with low iron storage are likely to benefit from iron therapy.

6. Although there is limited information on dopaminergic medications in children, small published reports show the effectiveness of levodopa and dopamine agonists in the management of RLS and PLMD.

\section{Research agenda}

1. Normative data on PLM index in children and adolescents.

2. Assessment of the cardiovascular and neurocognitive consequences of RLS and PLMD in children and adolescents.

3. Validated diagnostic and severity measures of pediatric RLS.

4. The role of other diagnostic modalities such as actigraphy and SIT (Suggested Immobilization Test) in the evaluation of RLS and PLMD in children.

5. The duration of iron therapy for responders and non-responders.

6. Long-term outcomes of pharmacologic therapy, especially the use of dopaminergic medications for management of RLS and PLMD in children and adolescents.

7. Iron metabolism in CNS of children with RLS and PLMD.

8. Peripheral metabolism of iron and metal receptors in children with RLS and PLMD.

\section{Acknowledgments}

DG is supported by National Institutes of Health grant HL65270, the Commonwealth of Kentucky Research Challenge for Excellence Trust Fund, and the Children's Foundation Endowment for Sleep Research. LKG is supported by an investigator initiated grant from Merck Company.

\section{References}

*The most important references are denoted by an asterisk. 
1. Chesson AL Jr, Anderson WM, Littner M, Davila D, Hartse K, Johnson S. Practice parameters for the treatment of restless legs syndrome and periodic limb movement disorder. An American Academy of Sleep Medicine Report. Standards of Practice Committee of the American Academy of Sleep Medicine. Sleep 1999;33:961-8. [PubMed: 10566915]

2. Montplaisir J, Boucher S, Poirier G, Lavigne, Lapierre O, Lesperance P. Clinical, polysomnographic, and genetic characteristics of restless legs syndrome: a study of 133 patients diagnosed with new standard criteria. Mov Disord 1997;12:61-5. [PubMed: 8990055]

3. Kotagal S, Silber MH. Childhood-onset restless legs syndrome. Ann Neurol 2004;56:803-7. [PubMed: 15505786]

4*. Muhle H, Neumann A, Lohmann-Hedrich K, Lohnau T, Lu Y, Winkler S. Childhood-onset restless legs syndrome: clinical and genetic features of 22 families. Mov Disord 2008;23:1113-21. [PubMed: 18381645]

5. Walters AS, Hickey K, Maltzman J, Verrico T, Joseph D, Hening W, et al. A questionnaire study of 138 patients with restless legs syndrome: the "Night-Walkers"' survey. Neurology 1996;46(1):925. [PubMed: 8559428]

6*. Picchietti D, Allen RP, Walters AS, Davidson JE, Myers A, Ferini-Strambi L. Restless legs syndrome: prevalence and impact in children and adolescents -the Peds REST Study. Pediatrics 2007;120:253-66. [PubMed: 17671050]

7*. Stefansson H, Rye DB, Hicks A, Petursson H, Ingason A, Thorgeirsson TE. A genetic risk factor for periodic limb movements in sleep. N Engl J Med 2007;357(7):639-47. [PubMed: 17634447]

8. Allen RP, Picchietti D, Hening WA, Trenkwalder C, Walters AS, Montplaisir J. Restless legs syndrome diagnosis and epidemiology workshop at the National Institutes of Health. Sleep Med 2003;4:101-19. [PubMed: 14592341]

9*. Picchietti D, Steven HE. Early manifestations of restless legs syndrome in childhood and adolescence. Sleep Med 2008;9:770-81. [PubMed: 18024165]

10*. Picchietti MA, Picchietti DL. Restless legs syndrome and periodic limb movement disorder in children and adolescents. Semin Pediatr Neurol 2008;15:91-9. [PubMed: 18555195]

11. Davis ID, Baron J, O'Riordan MA, Rosen CL. Sleep disturbances in pediatric dialysis patients. Pediatr Nephrol 2005;20:69-75. [PubMed: 15565279]

12. Trenkwalder C, Högl B, Benes H, Kohnen R. Augmentation in restless legs syndrome is associated with low ferritin. Sleep Med 2008;9(5):572-4. [PubMed: 17921065]

13. Earley CJ, Ponnuru P, Wang X, Patton SM, Conner JR, Beard JL, Taub DD, Allen RP. Altered iron metabolism in lymphocytes from subjects with restless legs syndrome. Sleep 2008;31(6):84752. [PubMed: 18548829]

14. Gamaldo CE, Earley CJ. Restless legs syndrome: a clinical update. Chest 2006;130:1596-604. [PubMed: 17099042]

15. Espinar-Sierra J, Vela-Buena A, Luque-Otero M. Periodic leg movements in sleep in essential hypertension. Psychiatry Clin Neurosci 1997;51:103-7. [PubMed: 9225372]

16. Pennestri MH, Montplaisir J, Colombo R, Lavigne G, Lanfranchi PA. Nocturnal blood pressure changes in patients with restless legs syndrome. Neurology 2007;68 (15):1213-8. [PubMed: 17420405]

17. Siddiqui F, Strus J, Ming X, Lee IA, Chokroverty S, Walters AS. Rise of blood pressure with periodic limb movements in sleep and wakefulness. Clin Neurophysiol 2007;118:1923-30. [PubMed: 17588809]

18. Pearson VE, Allen RP, Dean T, Gamaldo CE, Lesage SR, Early CJ. Cognitive deficits associated with restless legs syndrome (RLS). Sleep Med 2006;7:25-30. [PubMed: 16198145]

19. Wagner ML, Walters AS, Fisher BC. Symptoms of attention-deficit/hyperactivity disorder in adults with restless legs syndrome. Sleep 2004;27:1499-504. [PubMed: 15683140]

20. Picchietti DL, Walters AS. Restless legs syndrome and periodic limb movement disorder in children and adolescents: comorbidity with attention-deficit hyperactivity disorder. Child Adolesc Psychiatr Clin N Am 1996;5:729-40.

21. Picchietti DL, England SJ, Walters AS, Willis K, Verrico T. Periodic limb movement disorder and restless legs syndrome in children with attention-deficit hyperactivity disorder. J Child Neurol 1998;13:588-94. [PubMed: 9881529] 
22*. Picchietti DL, Underwood DJ, Farris WA, Walters AS, Shah MM, Dahl RE. Further studies on periodic limb movement disorder and restless legs syndrome in children with attention-deficit hyperactivity disorder. Mov Disord 1999;14:1000-7. [PubMed: 10584676]

23*. Chervin RD, Archbold KH, Dillon JE, Pituch KJ, Panahi P, Dahl RE. Association between symptoms of inattention, hyperactivity, restless legs, and periodic leg movements. Sleep 2002;25:213-8. [PubMed: 11902431]

24*. Cortese S, Konofal E, Lecendreux M, Arnulf I, Mouren MC, Darra F. Restless legs syndrome and attention-deficit/-hyperactivity disorder: a review of the literature. Sleep 2005;28:1007-13. [PubMed: 16218085]

25. Sadeh A, Pergamin L, Bar-Haim Y. Sleep in children with attention-deficit hyperactivity disorder: a meta-analysis of polysomnographic studies. Sleep Med Rev 2006;10:381-98. [PubMed: 16846743]

26. Sever Y, Ashkenazi A, Tyano S, Weizman A. Iron treatment in children with attention deficit hyperactivity disorder. A preliminary report. Neuropsychobiology 1997;35(4):178-80. [PubMed: 9246217]

27. Konofal E, Lecendreux M, Arnulf I, Mouren MC. Iron deficiency in children with attention-deficit/ hyperactivity disorder. Arch Pediatr Adolesc Med 2004 Dec;158(12):1113-5. [PubMed: 15583094]

28*. Konofal E, Lecendreux M, Deron J, Marchand M, Cortese S, Zaim M, Mouren MC, Arnulf I. Effects of iron supplementation on attention deficit hyperactivity disorder in children. Pediatr Neurol 2008 Jan;38(1):20-6. [PubMed: 18054688]

29. Oner P, Oner O. Relationship of ferritin to symptom ratings children with attention deficit hyperactivity disorder: effect of comorbidity. Child Psychiatry Hum Dev 2008 Sep;39(3):323-30. [PubMed: 18165896]

30. Cortese S, Lecendreux M, Bernardina BD, Mouren MC, Sbarbati A, Konofal E. Attention-deficit/ hyperactivity disorder, Tourette's syndrome, and restless legs syndrome: the iron hypothesis. Med Hypotheses 2008;70(6):1128-32. [PubMed: 18164140]

31. Picchietti DL, Walters AS. Moderate to severe periodic limb movement disorder in childhood and adolescence. Sleep 1999;22(3):297-300. [PubMed: 10341379]

32. Guilleminault C, Palombini L, Pelayo R, Chervin RD. Sleepwalking and sleep terrors in prepubertal children: what triggers them? Pediatrics 2003;111(1):e17-25. [PubMed: 12509590]

33*. Simakajornboon N, Gozal D, Vlasic V, Mack C, Sharon D, McGinley B. Periodic limb movement in sleep and iron status in children. Sleep 2003;26:735-8. [PubMed: 14572128]

34. Simakajornboon N, Kheirandish-Gozal L, Gozal D, Sharon D, Murry L, Abraham M. A long term follow-up study of periodic limb movement disorders in children after iron therapy. Sleep 2006;29(Suppl):A76.

35. Silber MH, Ehrenberg BL, Allen RP, Buchfuhrer MJ, Early CJ, Hening WA. An algorithm for the management of restless legs syndrome. Mayo Clin Proc 2004;79:916-22. [PubMed: 15244390]

36. Hening WA, Allen RP, Early CJ, Picchietti DL, Silber MH. An update on the dopaminergic treatment of restless legs syndrome and periodic limb movement disorder. Sleep 2004;27:970-99.

37. Molokwu OC. Appropriate use of dopamine agonists and levodopa in restless legs syndrome in an ambulatory care setting. Neurology 2008;42:627-32.

38. Schade R, Andersohn F, Suissa S, Haverkamp W, Garbe E. Dopamine agonists and the risk of cardiac valve regurgitation. N Engl J Med 2007;356(1):29-38. [PubMed: 17202453]

39. Allen RP, Earley CJ. Augmentation of the restless legs syndrome with carbidopa/levodopa. Sleep 1996;19:205-13. [PubMed: 8723377]

40. Earley CJ, Allen RP. Pergolide and carbidopa/levodopa treatment of the restless legs syndrome and periodic leg movements in sleep in a consecutive series of patients. Sleep 1996;19:801-10. [PubMed: 9085489]

41*. Walters AS, Mandelbaum DE, Lewin DS, Kugler S, England SJ, Miller M. Dopaminergic therapy in children with restless legs/periodic limb movements in sleep and ADHD. Dopaminergic Study Group. Pediatr Neurol 2000;22:182-6. [PubMed: 10734247] 
42. Konofal E, Arnulf I, Lecendreux M, Mouren MC. Ropinirole in a child with attention-deficit hyperactivity disorder and restless leg syndrome. Pediatr Neurol 2005;32:350-1. [PubMed: 15866437]

43. Martinez S, Guilleminault C. Periodic leg movements in prepubertal children with sleep disturbance. Dev Med Child Neurol 2004;46:765-70. [PubMed: 15540638]

44. Garcia-Borreguero D, Larrosa O, de la Liave Y, Verger K, Masramon X, Hernandez G. Treatment of restless legs syndrome with gabapentin: a double-blind, cross-over study. Neurology 2002;59:1573-9. [PubMed: 12451200]

45. Happe S, Sauter C, Kiösch G, Saletu B, Zeitlhofer J. Gabapentin versus ropinirole in the treatment of idiopathic restless legs syndrome. Neuropsychobiology 2003;48:82-6. [PubMed: 14504416]

46*. Newcorn JH, Schulz K, Harrison M, DeBellis MD, Udarbe JK, Halperin JM. Alpha 2 adrenergic agonists: neurochemistry, efficacy, and clinical guidelines for use in children. Pediatr Clin North Am 1998;45:1099-122. [PubMed: 9884677]

47. Bassetti CL, Mauerhofer D, Gugger M, Mathis J, Hess CW. Restless legs syndrome: a clinical study of 55 patients. Eur Neurol 2001;45(2):67-74. [PubMed: 11244268]

48. Winkelmann J, Wetter TC, Collado-Seidel V, Gasser T, Dichgans M, Yassouridis A, et al. Clinical characteristics and frequency of the hereditary restless legs syndrome in a population of 300 patients. Sleep 2000;23(5):597-602. [PubMed: 10947027]

49. O’Brien LM, Holbrook CR, Faye Jones V, Gozal D. Ethnic difference in periodic limb movements in children. Sleep Med 2007;8(3):240-6. [PubMed: 17368099]

50. Crabtree VM, Ivanenko A, O’Brien LM, Gozal D. Periodic limb movement disorder of sleep in children. J Sleep Res 2003;12(1):73-81. [PubMed: 12603789] 
Table 1

Criteria for making the diagnosis of definite RLS in children. ${ }^{6}$

1. The child meets all four essential adult criteria for RLS (the urge to move the legs, is worse during rest, relived by movement and worse during the evening and at night) and

2. The child relates a description in his or her own words that is consistent with leg discomfort (the child may use terms such as oowies, tickle, spiders, boo-boos, want to run and a lot of energy in my legs to describe the symptoms. Age-appropriate descriptors are encouraged)

Or

1. The child meets all four essential adult criteria for RLS and

2. Two of the three following supportive criteria are present.

a. Sleep disturbance for age

b. A biologic parent or sibling has definite RLS

c. The child has a polysomnographically documented periodic limb movement index of 5 or more per hour of sleep. 
Table 2

Criteria for making the diagnosis of probable RLS in children. ${ }^{6}$

1. The child meets all four essential adult criteria for RLS except criterion\# 4 (the urge to move or sensations are worse in the evening or at night than during the day), and

2. The child has a biologic parent or sibling with definite RLS.

Or

1. The child is observed to have behavior manifestations of lower extremity discomfort when sitting or lying, accompanied by motor movement of the affected limbs, the discomfort has characteristic of adult criteria 2, 3, and 4 (i.e., is worse during rest and inactivity, relieved by movement, and worse during the evening and at night) and

2. The child has a biologic parent or sibling with definite RLS 
Table 3

Criteria for making the diagnosis of possible RLS in children. ${ }^{6}$

1 The child has periodic limb movement disorder and

2 The child has a biologic parent or sibling with definite RLS, but the child does not meet definite or probable childhood RLS definitions. 


\section{Table 4}

Criteria for making the diagnosis of PLMD in children. ${ }^{6}$

1 Polysomnographic study shows a PLM index of $>5 / h$ and

2 Clinical sleep disturbance for age must be evident as manifested by sleep-onset problems, sleep-maintenance problems, or excessive daytime sleepiness and

3 The leg movements cannot be accounted for by sleep-disordered breathing or medication effect (antidepressant medication). 